\title{
Food allergy: Two case reports and management challenges in a resource-limited setting
}

\author{
O F Adeniyi, ${ }^{1}$ MBBS, FMC (Paed), MSc (Child Health); J K Renner, ${ }^{2}$ MBBS, FWACP \\ ${ }^{1}$ Department of Paediatrics, College of Medicine, University of Lagos; and Lagos University Teaching Hospital, Lagos, Nigeria \\ ${ }^{2}$ Department of Paediatrics, College of Medicine, Babcock University, Sagamu, Ogun State, Nigeria
}

Corresponding author: OF Adeniyi (layo_funke@yahoo.co.uk)

\begin{abstract}
Background. Food allergy has been well described in white children, and cow's milk protein allergy (CMPA) still remains the most common allergy in these children. Information on the same subject in developing countries is very limited, and management of this condition remains challenging.

Case presentation. We report on two cases of children with multiple food allergies. The first patient presented with chronic diarrhoea following the introduction and use of several infant formulas, while the second patient had more severe allergic reactions following ingestion of milk, egg and wheat. Elimination of identified triggers from the diet resulted in significant clinical recovery in both cases. Conclusion. Food allergy, especially CMPA, should be considered more frequently in infants and children from developing countries, especially when there is a significant reaction to the introduction of cow's milk. A high index of suspicion and appropriate laboratory support are also needed in the diagnosis and management of other food allergies in the African setting.
\end{abstract}

S Afr J Child Health 2015;9(3):103-104. DOI:10.7196/SAJCH.8152

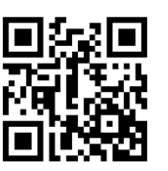

Multiple food allergies have been well described in developed countries; making a diagnosis and subsequent management in these countries where the facilities are readily available is relatively easy. ${ }^{[1-3]}$ Cow's milk protein allergy (CMPA) remains the most common food allergy in these countries. ${ }^{[2,3]}$ Many primary care physicians in developing countries are still unaware of the condition, especially in sub-Saharan Africa, where it may be misdiagnosed as food poisoning, infective diarrhoea or even lactose intolerance. Most children with vomiting, diarrhoea or other foodrelated symptoms are given antibiotics either at home or by some physicians before being referred for any specialist care. In many developing countries, there is a paucity of data on food allergy, and diagnosis and management of this condition is still very challenging due to its variable clinical presentation.

We report two cases of children with multiple food allergies, with variable clinical presentation, and highlight the challenges in managing cases of food allergy in the African setting.

\section{Case presentation 1}

OC, a female infant, was first seen at the age of 8 months on account of recurrent loose stools for 2 months. Stools were non-mucoid, but there was a history of occasional bloody streaks in the stools. There was no associated vomiting or fever. Each episode of loose stools was precipitated by ingestion of formula milk feeds. Infant formula was introduced initially at the age of 1 month, but the patient had a similar reaction to the use of three different formulas. There was also a similar reaction to ingestion of eggs. She had a course of metronidazole with each episode of the bloody stools, with no improvement.

An initial diagnosis of lactose intolerance was suggested to the mother (this was not confirmed by laboratory tests) and thus at the age of 5 months, a soy-based formula was introduced; however, there was no appreciable improvement of symptoms. At presentation, the patient was on fortified maize cereal with soy-based milk, peanuts and margarine, and mashed potatoes with fish.

The rest of the history was unremarkable except for allergic rhinitis in the mother, atopical dermatitis and asthma in the maternal uncle and allergic conjunctivitis in the paternal grandmother. The patient was the only child of a monogamous family.

Physical examination was normal and anthropometry was as follows: weight $7.2 \mathrm{~kg}$ (15th percentile), length $68 \mathrm{~cm}$ (50th percentile) and occipito frontal circumference $45 \mathrm{~cm}$ (85th percentile).

A tentative diagnosis of CMPA was made. Investigations requested, namely skin prick tests and IgE levels, were not done owing to financial constraints and unavailability of the reagents for the skin prick tests in the hospital at the time.

Cow's milk was excluded from the diet and an extensively hydrolysed formula (EHF) preparation (Pepti Junior, Cow \& Gate, UK) was subsequently introduced. After about 2 weeks, the passage of loose stools and blood-streaked stools resolved. The child thrived subsequently and follow-up by the 2nd birthday showed that the patient weighed $12 \mathrm{~kg}$. Following the 2 nd birthday i.e. the $3 \mathrm{rd}$ year of life, the EHF was discontinued and cow's milk was gradually introduced into the diet. Symptoms never reoccurred and she currently eats all food, including eggs.

\section{Case presentation 2}

The second case, JI, a 16-month-old female child, was referred from a private children's hospital on account of allergic reaction to infant formula and eggs.

She was the product of a normal, full-term pregnancy and weighed $3.3 \mathrm{~kg}$ at birth. On the first day of life, JI was given Nan (Nestlé, Australia) formula as the mother was not yet lactating well, but she developed generalised body rash and the formula was discontinued. The mother was able to exclusively breastfeed for 2 months, and by the 3rd month of age another infant formula was introduced (SMA Gold, SMA, UK); however, again she developed a body rash, and facial and abdominal swelling. Subsequently, she had similar reactions when eggs, fish and wheat were introduced, and had recurrent reactions whenever she was given any of these foods. The mother had also noticed that she was gaining weight poorly. A month prior to presentation, she developed vomiting, diarrhoea and abdominal distension with excessive flatulence within 2 hours of feeding. The mother had given metronidazole and ampiclox before presentation at the hospital. The patient's paternal uncle was a known asthmatic. 
Significant findings on physical examination revealed a malnourished child, pale with sparse, fluffy hair, with no oedema. Her weight was $7.1 \mathrm{~kg}$ which is $<5$ th percentile and length $68 \mathrm{~cm}$, which was also $<5$ th percentile. Weight for length was similarly $<5$ th percentile. Systemic examination was normal.

An assessment of failure to thrive secondary to food allergy (CMPA) to rule out gluten sensitivity was made. The results of the investigation revealed that the radio-allergosorbent test (RAST) was positive for egg white f1, milk f2, codfish f3, wheat f4, peanut f13 and soya bean $\mathrm{f} 14$. The tissue trans glutaminase (TTG) antibody test and endomysial antibodies (TTG IgA-0.30(0-10), TTG IgG-0.9(0$10)$; endomysium $\operatorname{IgA}$ ) were negative. Serum proteins were within normal limits.

Cow's milk was eliminated from the diet and an EHF was introduced, but the patient did not tolerate this. She ultimately tolerated an amino acid-based formula (Neocate Active, Nutricia, UK) courtesy of some relatives in the UK and USA who were able to help procure the formula. However, this was an expensive therapy as each pack of this formula cost about NGN20 000 (USD120) and the recommendation is for at least two packs/day. Within a month of commencement of the formula, the patient's symptoms resolved. Eggs, wheat and fish were then reintroduced, which she now tolerates. However, at 24 months of age when cow's milk was reintroduced, she still reacted to it and therefore she is currently still on Neocate formula with rational use. She is also receiving the recommended daily allowance for supplements, namely vitamins A, $\mathrm{D}$ and $\mathrm{B}$ complex and calcium, and during follow-up visits she has been observed to be thriving.

\section{Discussion}

In our first case diagnosis of possible infantile CMPA was made based solely on history, physical examination and the positive response to a dietary elimination trial. The diagnostic workup was significantly limited by the financial constraints of the parents, unwillingness to carry out other confirmatory tests and unavailability of local resources for the tests. A more thorough laboratory work-up a with skin prick test, RAST and IgE tests could have supported the diagnosis more firmly. ${ }^{[4-6]}$ Nevertheless, the patient still had significant symptoms suggestive of the condition; it has been observed in some studies that $18 \%$ of children with blood in their stools had CMPA. ${ }^{[7]}$ The failure of improvement of symptoms following the use of soy-based formula is not surprising as up to $10-14 \%$ of CMPA patients also cross-react to soy protein, especially infants $<6$ months of age. ${ }^{[8.9]}$ The use of antibiotics in many developing countries remains questionable in patients who present with gastroenteritis. This is a result of lack of appropriate laboratory support in making a diagnosis, especially in rural areas. It appears that many cases of vomiting or diarrhoea, which may occur as a symptom of food allergy, are believed to be of infective origin and thus children have often had a course of antibiotics before presentation at hospital.

The second case illustrates a more severe clinical manifestation of food allergy, which has been reported by other authors. ${ }^{[10]}$ Although the confirmatory tests were done in this instance because of willing parents, the affordability and sustainability of the amino acid formula has been very challenging to the family. This demonstrates the plight of average citizens in the African setting who may not be able to afford such an expensive therapy for their children. These two cases highlight the variable presentation of multiple food allergies. The reason for allergy to multiple foods may be explained by possible cross-reactivity of the allergens. ${ }^{[10]}$ In most cases, the children become desensitised by the age of 2 years and most will outgrow the allergies, as occurred with the first case in this study. However, there lies the possibility of development of other allergic conditions, namely allergic conjunctivitis, rhinitis or asthma in the future and therefore the parents were duly counselled about this.

Challenges in the management of food allergy in the African setting are numerous, namely misdiagnosis, late presentation, and unavailability of diagnostic facilities and appropriate substitution diet. Therefore, a high index of suspicion is important in resourcelimited countries where confirmatory tests cannot be carried out. Eliminating the offending food from the diet and subsequent resolution of symptoms can help to confirm the diagnosis.

It has been suggested that in tribes/ethnic groups where cow's milk is given to young infants for nutritional purposes or cultural reasons and the children develop gastrointestinal symptoms or other clinical manifestation, the possibility of CMPA should be entertained. ${ }^{[11]}$ Currently, it has been suggested that a supervised food challenge be performed after a few weeks of elimination diet and gradual reintroduction be done between 9 months and 18 months of age depending on the severity of the allergic reaction. ${ }^{[10,11]}$

\section{Conclusion}

Clinicians working in resource-limited countries need to be aware of and have a high index of suspicion of food allergy, especially CMPA, when the history and clinical findings are suggestive. A therapeutic elimination diet should be commenced, and substitution therapy with the appropriate formula in the case of CMPA, if affordable, should be instituted.

\section{References}

1. Minford AMB, Macdonald A, Littlewood JM. Food intolerance and food allergy in children: A review of 68 cases. Arch Dis Child 1982;57(10):742-747.

2. Sicherer SH. Epidemiology of food allergy. J Allergy Clin Immunol 2011;127(3):594-602. [http://dx.doi.org/10.1016/j.jaci.2010.11.044]

3. Rona RJ, Keil T, Summers C, et al. The prevalence of food allergy: A metaanalysis. J Allergy Clin Immunol 2007;120(3):638-646. [http://dx.doi. org/10.1016/j.jaci.2007.05.026]

4. Huang J, Walker WA. Review of pediatric gastrointestinal disease and nutrition. London: BC Decker, 2005

5. Vandenplas Y, Koletzko S, Isolauri E, et al. Guidelines for the diagnosis and management of cow's milk protein allergy in infants. Arch Dis Child 2007;92(10):902-908. [http://dx.doi.org/10.1136/adc.2006.110999]

6. Eigenmann PA. The spectrum of cow's milk allergy. Pediatr Allergy Immunol 2007;18(3):265-271. [http://dx.doi.org/10.1111/j.1399-3038.2006.00528.x]

7. Arvola T, Ruuska T, Keränen J, Hyöty H, Salminen S, Isolauri E. Rectal bleeding in infancy: Clinical, allergological, and microbiological examination. Pediatrics 2006;117(4):760-768. [http://dx.doi.org/10.1542/peds.2005-1069]

8. Klemola T, Vanto T, Juntunen-Backman K, et al. Allergy to soy formula and to extensively hydrolyzed whey formula in infants with cow's milk allergy: A prospective, randomized study with a follow-up to the age of 2 years. J Pediatr 2002;140(@):219-224. [http://dx.doi.org/10.1067/mpd.2002.121935]

9. Zeiger RS, Sampson HA, Bock SA, et al. Soy allergy in infants and children with IgE-associated cow's milk allergy. J Pediatr 1999;134(5):614-622.

10. Koletzko S, Niggemann YB, Arato ZA, et al. Diagnostic approach and management of cow's-milk protein allergy in infants and children: ESPGHAN GI Committee practical guidelines. J Pediatr Gastroenterol Nutr 2012;55(2):221229. [http://dx.doi.org/10.1097/MPG.0b013e31825c9482]

11. Krüger C, Malleyeck I. Diagnosing possible infantile cow's milk protein allergy in rural Africa, when history and physical examination are the only tools: A case report. Cases J 2009;2:6287. [http://dx.doi.org/10.4076/1757-1626-2-6287] 